\title{
Fürsorge für die Geisteskranken außerhalb der Anstalten ${ }^{1}$ ).
}

\author{
Von
}

\author{
E. Meyer, Königsberg i. Pr.
}

(Eingegangen am 4. Mai 1922.)

In der letzten Zeit ist die Frage eines Reichsirrengesetzes wieder viel erörtert, ausgehend besonders von der Idee des angeblich unzureichenden Rechtsschutzes der Geisteskranken. Zu einem Gesetz mit solcher Tendenz geben meines Erachtens die tatsächlichen Verhältnisse keinen Anlaß, dagegen weit eher zu einem, das die Versorgung der Geisteskranken außerhalb der Anstalten regeln würde.

In den früheren guten Zeiten kam das bei uns verhältnismäßig wenig in Betracht. Wir waren mit Recht stolz darauf, daß wir mehr und mehr die Geisteskranken in mustergültigen Anstalten unterbringen konnten, so daß das Bedürfnis zur Verpflegung und Versorgung von Kranken in anderer Weise jedenfalls nicht dringend erschien.

Die koloniale und familiäre Pflege der Geisteskranken hat mit aus diesem Grunde in Deutschland, von einzelnen Landesstrichen abgesehen, keinen größeren Umfang erreicht.

Die Not der Gegenwart hat das von Grund aus geändert. Nach jeder Richtung hin suchen die bedrängten Kommunen sich zu entlasten, und dabei fordert auch der früher wenig belangreiche Kostenunterschied zwischen den Unterhaltungskosten in und außerhalb der Anstalt besondere Beachtung, und ebenso jede Möglichkeit, die Kranken ihr Brot, und sei es auch nur teilweise, erwerben zu lassen, was früher ebenfalls nicht so ins Gewicht fiel, um so mehr, als die Kranken, die draußen doch oft mit Schwierigkeiten kämpften, gute Arbeitskräfte im Rahmen der Anstalten abgaben. Auch heute muß das Wohl der Kranken unser oberstes Gebot bleiben, aber, soweit dieses es gestattet, müssen wir Mittel und Wege zu finden suchen, möglichst viele unserer Kranken außerhalb der Anstalten zu belassen. Es handelt sich dabei, wie ich einfügen will, naturgemäß nur um sogenannte arme Kranke, d. h. solche, die aus öffentlichen Mitteln ganz oder teilweise erhalten werden müssen. Bei der chronischen Natur eines großes Teils der Geisteskrankheiten ist die

1) Vortrag, gehalten auf der Jahresversammlung des Nordostdeutschen Vereins f. Psych. u. Neurol. 22. IV. 1922. 
Zahl der Kranken, die früher oder später den Gemeinden zur Last fallen, aber eine große, heute, wie wir alle wissen, mehr denn je.

Andere Länder, England und vor allem Schottland, sind schon lange bestrebt, möglichst viele Geisteskranke außerhalb der Anstalten zu verpflegen.Wenn auch die Gesamtorganisation für die Beaufsichtigung der Geisteskranken, die diese Länder haben, so mustergültig sie in vieler Richtung ist, in unserer gegenwärtigen Lage nicht als Vorbild dienen kann, so ist doch manches daraus sehr lehrreich: Nach dem Gesetz wird in Schottland jeder arme Kranke der Zentralbehörde für Geisteskranke gemeldet. Nach dem Berichte dieser befanden sich im Jahre 1908/09 13600 arme Kranke in Anstalten und 2900 in Privatpflege, also mehr als ein Fünftel der Gesamtzahl. Diese waren zum kleineren Teil bei ihren Angehörigen, zum größeren bei Fremden untergebracht. Alle diese Kranken werden von der Zentralbehörde beaufsichtigt und zwar werden sie durch deren ärztliche Mitglieder, langjährige Psychiater von Ruf, mindestens einmal im Jahre untersucht. Im übrigen werden sie versorgt von dem Arzte und dem Beamten der Armenverwaltung des betreffenden Distriktes 1 ).

In Deutschland besteht demgegenüber bisher im wesentlichen eine polizeiliche Aufsicht, deren Träger die Kreisärzte im Verein mit den Polizeiorganen sind. $\mathrm{Zu}$ einer eigentlichen Fürsorge sind in letzter Zeit erst Ansätze gemacht, so besonders in Frankfurt a. M. durch Raecke $^{2}$ ) und in Plauen, wo nach dem freundlichen Bericht von Herrn Kollegen Schwabe sehr eifrig gearbeitet wird. Auch in RheinlandWestfalen sind ähnliche Bestrebungen im Gange. Schon lange beschäftigen sich ferner die "Hilfsvereine" mit solchen Aufgaben, ihre Mittel sind aber gegenwärtig zu gering, um wesentliche Leistungen zu vollbringen.

Zweckmäßig erscheint es mir, einen möglichst großen Bezirk, also in unserem Falle die ganze gegenwärtige Provinz Ostpreußen, zu einheitticher Fürsorgetätigkeit zu vereinigen. Diese würden die örtlichen Instanzen einmal ausüben, die Kreis- und Stadtverwaltungen und die betreffenden beamteten Ärzte, und dann eine Oberaufsichtsbehörde, deren Träger Psychiater mit mehrjähriger Erfahrung und Schulung sein müßten, wie sie an den Anstalten und der Klinik zu Gebote stehen. Der administrative Teil läge am besten in den Händen der staatlichen oder kommunalen Verwaltung der Provinz. Eine Oberaufsicht, eine Art Zentralbehörde, scheint mir geboten, da es sonst vielfach an der nötigen Anregung zur Durchführung und Förderung der Fürsorge fehlen würde.

i) Vgl. E. Meyer, Fürsorge für Geisteskranke in England und Schottland. Arch. f. Psychiatr. u. Nervenkrankh. 39, H. l.

2) Vgl. dessen Aufsätze über ,,soziale Psychiatrie". 
Diesen zentralen wie den lokalen Fürsorgeämtern würden die Kranken bei der Entlassung aus den Anstalten gemeldet, zweckmäßigerweise nicht nur die armen Kranken, sondern auch - zur Orientierung für alle Fälle die Selbstzahler, wenn auch allein die ersteren, soweit möchte ich zur Zeit in meinen Vorschlägen nur gehen, Gegenstand der Fürsorge und Aufsicht sein würden. Ob diese Versorgung von vornherein eine aktive oder eine mehr abwartende sein sollte, darïber müßte die Erfahrung entscheiden. Mir würde das Einsetzen der Fürsorge schon von Anfang an, natürlich in diskreter Weise, richtig erscheinen, unerläßlich dann, sobald Unterstützung aus öfentlichen Mitteln beansprucht würde. Je früher eine Versorgung versucht werden kann, um so sicherer wird es vor allen Dingen gelingen, geeignete Beschäftigung für die Kranken aufzufinden, was ja mit zu den Hauptaufgaben der lokalen Fürsorgeämter gehören sollte. ${ }^{1}$ )

Neben den Kranken, die bei der Entlassung aus den Anstalten zur Kenntnis der Fürsorgeämter kämen, könnten auch die mehr und mehr registriert und versorgt werden, die noch nicht in Anstalten waren, aber schon öffentliche Mittel in Anspruch zu nehmen genötigt sind.

Unter polizeilicher Aufsicht, die bei uns zur Zeit ja allein offiziell besteht, die aber naturgemäß eine eigentliche Fürsorge nicht sein kann, könnten die Kranken, die als gemeingefährlich in die Anstalten ursprünglich eingewiesen waren, wohl zweckmäßig verbleiben, ohne daß sie darum der Versorgung im eigentlichen Sinne verlustig gehen würden.

Das ist in ganz kurzen Zügen die Fürsorge für die Geisteskranken außerhalb der Anstalten, wie sie mir vorschwebt. Die Einzelheiten können sich nur aus der weiteren gemeinsamen Besprechung, Durcharbeitung: und schließlich der Erfahrung ergeben.

Die Kosten für die Einrichtung der Fürsorge würden sicher durch die Ersparnisse an Anstaltsverpflegung eingebracht werden. Eine Beteiligung der Landesversicherungsanstalten und auch der Krankemkassen wäre gewiß zu erreichen.

Angaben über die Höhe der Ausgaben, die ich aber nach den Mitteilungen von anderen Stellen als nicht sehr wesentlich veranschlagen möchte, sind noch nicht möglich, ebensowenig wie ich über die Zahl der in Betracht kommenden Kranken schon etwas sagen kann. Ich bin aber überzeugt, daß sie sicher zahlreich genug sind, um die aufgewandte Mübe zu Iohnen.

Der Fürsorge für die Psychopathen habe ich bisher nicht gedacht. Sie wird, wie auch die Berichte aus Frankfurt a. M. und Planen zeigen, vielfach mit der für die Geisteskranken zusammenfallen, um so mehr, da die Fälle, wo eine Abgrenzung von psychopathischer Konstitution

1) Auf die Einzelheiten der Einrichtung dieser Fürsorgeämter nsw. kann hier nicht eingegangen werden. 
476 E. Meyer: Fursorge für die Geisteskranken außerhalb der Anstalten.

und eigentlicher Psychose, insbesondere Dementia praecox, sehr schwierig ist, ja nur zu häufig sind.

Zum Schluß möchte ich noch auf eine Möglichkeit der Verpflegung außerhalb der eigentlichen Anstalten hinweisen, die freilich etwas aus dem Rahmen meines Themas fällt. Ich muß dabei wieder auf das Beispiel von Schottland hinweisen. Dort werden nämlich Geisteskranke, deren Leiden in ein im wesentlichen gleichmäßig ruhiges Stadium gekommen ist, und die somit der Anstaltsbehandlung im strengen Sinne nicht mehr bedürfen, aber doch einer gewissen dauernden Aufsicht nicht entbehren können, in eigens dafür bestimmten und entsprechend eingerichteten Sälen von Armenhäusern verpflegt, wohlgemerkt unter psychiatrischer Oberaufsicht.

Ich würde in der gleichen Einrichtung bei uns keinen Rückschritt sehen, sondern nur ein Eingehen auf das Gebot der Stunde ${ }^{1}$ ).

1) Vgl. aJlgemein: Möli, Fürsorge für Geisteskranke. Halle 1915. 